Nervenarzt 2022 $\cdot 93: 111-113$

https://doi.org/10.1007/s00115-021-01250-6

Angenommen: 8. Dezember 2021

(C) The Author(s), under exclusive licence to Springer Medizin Verlag GmbH, ein Teil von Springer Nature 2022

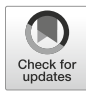

Aus Gründen der besseren Lesbarkeit wird in diesem Beitrag überwiegend das generische Maskulinum verwendet. Dies impliziert immer beide Formen, schließt also die weibliche Form mitein.

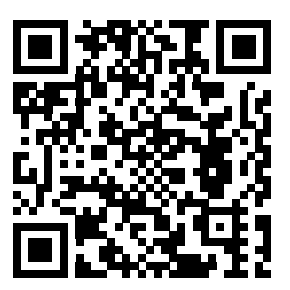

QR-Code scannen \& Beitrag online lesen

\title{
Neuropädiatrie trifft Neurologie
}

\section{Gemeinsamkeiten - Unterschiede?}

\author{
Ulrike Schara-Schmidt \\ Abteilung Neuropädiatrie, Entwicklungsneurologie und Sozialpädiatrie, Klinik für Kinderheilkunde I, \\ Universitätsklinikum Essen (AöR), Essen, Deutschland
}

Sehr geehrte Leserinnen und Leser,

ich freue mich, Ihnen mit der heutigen Ausgabe von Der Nervenarzt Beiträge zu wichtigen Aspekten in der frühen Diagnose, der Diagnostik und der Therapie pädiatrischer neurologischer Krankheitsbilder vorstellen zu dürfen. Diese Krankheiten können im Kindesalter, in der Neugeborenenzeit oder gar pränatal beginnen. Eine bessere Versorgung und Therapie können ihren Verlauf deutlich beeinflussen, sodass die Patienten heute das Erwachsenenalter erreichen.

\section{》) Krankheitsbilder präsentieren sich über die gesamte Lebensspanne häufig unterschiedlich}

Die Krankheitsbilder, die sich zeitlich über die gesamte Lebensspanne manifestieren und sich dabei durchaus unterschiedlich präsentieren, sind eine Herausforderung für die neurologischen Fachdisziplinen. Die neuen diagnostischen und therapeutischen Verfahren sind vom Kindes- bis ins Erwachsenenalter übergreifend einsetzbar und können aus der Neuropädiatrie kommend über die gesamte Lebensspanne sinnvoll angewandt werden. Daher heißt das übergeordnete Thema dieser Ausgabe „Neuropädiatrie trifft Neurologie”.

Die Neuropädiatrie wird in ihren diagnostischen und therapeutischen Möglichkeiten komplexer; sie wird bei einzelnen Erkrankungen eine Vorreiterrolle für den Einsatz komplexer Therapien mit allen Konsequenzen, von der Veränderung des Lebens der Patienten mit ihren Familien bis hin zur Umstrukturierung der Versorgungsleistung, einnehmen. Für die Patienten bedeutet dies oft eine deutliche Ver- besserung ihres Krankheitsverlaufes, d.h. auch eine verlängerte Lebenserwartung und eine verbesserte Lebensqualität.

Neurologische Erkrankungen betreffen ca. 3-5\% aller Kinder und nehmen bei Erwachsenen zu. Insbesondere in der alternden Population Westeuropas sind sie neben Herz-Kreislauf- und Tumorerkrankungen die häufigste Ursache für Morbidität und Mortalität ein.

Wir möchten mit dieser Ausgabe anhand einzelner Erkrankungen bzw. diagnostischer Vorgehensweisen auf eine verbesserte Diagnostik, auf optimierbare Algorithmen zur Früherkennung, z. B. des kindlichen Schlaganfalls oder der jetzt auch behandelbaren 5q-SMA, hinweisen. Die Autoimmunerkrankungen des zentralen Nervensystems sowie schwere Epilepsien, die einer epilepsiechirurgischen Intervention, z. B. einer Hemisphärotomie bedürfen, profitieren von einer frühen Diagnose und einer entsprechend früh einsetzenden Therapie.

\section{》) Die Kombination von}

Genomik und Proteomik hilft, pathophysiologische Abläufe besser zu verstehen

Gangfuß et al. beschreiben anhand von Genomik und Proteomik am Beispiel neuromuskulärer Erkrankungen als Paradigma eine Kombination der auf "Next-generation-sequencing"-Analyse basierten DNAUntersuchung als Omics-Technologie und der Integration von Proteinanalysen (Proteomik). Besonderes Augenmerk liegt auf der Kombination von Genomik und Proteomik, die zum einen der Erforschung neuer Erkrankungen dienen kann, zum anderen aber auch helfen kann, pathophysiologi- 
sche Abläufe besser zu verstehen. Dies ist ausgehend von neuromuskulären Erkrankungen im Kindes- und Jugendalter durchaus ins Erwachsenenalter und auf andere neurologische Erkrankungen zu übertragen.

Zum Thema Diagnostik beschreiben Kölbel et al. das Neugeborenenscreening für die $5 q$-spinale Muskelatrophie (5qSMA). Durch die Entwicklung und Zulassung medikamentöser Therapieoptionen für eine bis noch vor einem Jahrzehnt unbehandelbare Erkrankung mit z.T. sehr verkürzter Lebenserwartung stehen heute drei medikamentöse Therapien zur Verfügung, und das nicht nur für Kinder, sondern auch für Erwachsene. Durch diese Entwicklung hat sich auch in der Neurologie die Therapie der erwachsenen Patienten mit 5q-SMA entscheidend verändert. Die Phänotypen ändern sich unter der Therapie und wir werden mehr Menschen mit diesen Erkrankungen sowohl in der Neuropädiatrie als auch später in der Neurologie behandeln können und gleichsam werden mehr Patienten mit einer milderen Form der 5q-SMA in der Neurologie diagnostiziert und therapiert werden können.

\section{I) Individuelle Unterschiede im Ansprechen auf Medikamente rücken in den Fokus}

Ziegler beschreibt in seinem Artikel zur Präzisionsmedizin wesentliche Aspekte zum Einsatz moderner diagnostischer Methoden und damit zu verbesserten diagnostischen Möglichkeiten, auch zu maßgeschneiderten Krankheitspräventionen und zu einer maßgeschneiderten Therapie. Das ist eine wesentliche Veränderung in der Neuropädiatrie, in der in der Vergangenheit die Behandlungen häufig nur symptomatisch sein konnten. Individuelle Unterschiede im Ansprechen und in der Verträglichkeit der Medikamente wurden und werden häufig auch noch nicht berücksichtigt. Das ist ein Vorgehen, das immer breiter verfolgt wird von neuromuskulären Erkrankungen über neurometabolische Erkrankungen bis hin zu Epilepsien und weiteren Erkrankungen. Auch dies wird nicht auf der Neuropädiatrie begrenzt bleiben, sondern diese Vorgehensweisen werden auch in der
Neurologie angewandt und weiterentwickelt werden.

Nicht immer ist die medikamentöse Therapie ausreichend. So beschreiben Hartlieb et al. die Hemisphärotomie in der pädiatrischen Epilepsiechirurgie. Hemisphärisch epileptogene Läsionen führen meist zu früh beginnenden, schwierig verlaufenden Epilepsien mit einer hohen Anfallsfrequenz, die nicht gut medikamentös beherrschbar sind. Hemisphärotomien stellen mit 16-21\% einen Großteil der epilepsiechirurgischen Eingriffe im Kindesalter dar. Hier konnten die Autoren zeigen, dass die Operationen sowohl in Bezug auf epileptologische Aspekte als auch auf zu erwartende funktionelle Konsequenzen eine große Rolle spielen. Durch Langzeitbeobachtung konnten die Autoren zeigen, dass möglichst viel neoplastisches Potenzial der gesunden Hemisphäre genutzt werden kann, wenn eine Operation frühzeitig erfolgt.

Das Thema Autoimmunerkrankung des zentralen Nervensystems, hier vor allem die Multiple Sklerose, ist Gegenstand des Artikels von Rostásy et al. Autoimmunerkrankungen, die das zentrale Nervensystem betreffen, sind in der Erwachsenenmedizin relativ häufige Erkrankungen. Bei Kindern und Jugendlichen rücken sie hingegen zunehmend erst jetzt in den Fokus der Aufmerksamkeit. Es sind in den letzten Jahren wichtige Fortschritte in der Therapie in dieser Altersgruppe erzielt worden, die zu einer besseren Charakterisierung und Behandlung der Erkrankung geführt haben. Dennoch sind wir noch weit entfernt von den Möglichkeiten, gerade der medikamentösen Therapien, die in der Neurologie zur Verfügung stehen. Auch andere autoantikörpervermittelte Enzephalitiden, wie z.B. NMDA(N-Methyl-D-Aspartat)-Rezeptor- oder MyelinOligodrendozyten-Glycoprotein(MOG)Enzephalitiden, sowie die MOG-Antikörper-assoziierten Erkrankungen werden beschrieben. Diese Erkrankungen treten zunehmend im Kindes- und Jugendalter auf bzw. werden häufiger diagnostiziert. Auch hier gilt, je eher die Diagnose und je eher die Therapie beginnt, desto besser ist das Outcome.

Damit ist auch das Schlagwort für den kindlichen Schlaganfall gefallen: "time is brain“. Hier sind die optimierten Algorith- men im Erwachsenenalter bekannt, aber noch nicht flächendeckend auf Kinder und Jugendliche übertragbar. Es fehlt noch an der Umsetzung von Früherkennung, rascher und adäquater intensivmedizinischer Versorgung und umgehender interventioneller Therapie. So konnten Gerstl et al. zeigen, dass der arteriell ischämische Schlaganfall im Kindes- und Jugendalter zu den zeitkritischsten Notfällen in der Pädiatrie gehört, aber häufig mit einer oft prognostisch relevanten Zeitverzögerung diagnostiziert wird. Die Autoren stellen sehr fokussiert die relevanten Gründe für diese Diskrepanz dar und diskutieren notwendige Verbesserungen dieser Situation.

\section{॥) Der arteriell ischämische Schlaganfall gehört zu den zeitkritischsten Notfällen in der Pädiatrie}

Anhand der in dieser Ausgabe von Der Nervenarzt zusammengestellten Manuskripte können übergeordnete Aussagen für Neuropädiatrie sowie Neurologie getroffen werden und sie sind deshalb auch für beide Fachdisziplinen wichtig. Fortschritt in der Diagnostikführt zur frühen Diagnose und damit verbesserter Therapiesituation. "Time is brain and motoneuron" - und das über die gesamte Lebensspanne! Zunehmend werden Krankheitsbilder in der Neuropädiatrie als multidisziplinär begriffen und bei möglichen medikamentösen oder auch operativen Methoden wird klar, dass, je eher die adäquate Therapie erfolgt, desto besser ist das Outcome. Zuletzt ist in der Multidisziplinarität die Präzisionstherapie für den einzelnen Menschen das Wichtige. Nur so sind wir gewappnet für eine gute Transition unserer Patienten.

Das Kind ist kein kleiner Erwachsener, Unterschiede gibt es sicher, aber bei näherem Hinschauen auch viele Gemeinsamkeiten!

In diesem Sinne wünsche ich viel Freude bei der Lektüre.

Univ.-Prof. Dr. med. U. Schara-Schmidt 


\section{Korrespondenzadresse}

Univ.-Prof. Dr. med. Ulrike Schara-Schmidt Abteilung Neuropädiatrie, Entwicklungsneurologie und Sozialpädiatrie, Klinik für Kinderheilkunde I, Universitätsklinikum Essen (AöR)

Hufelandstr. 55, 45147 Essen, Deutschland ulrike.schara-schmidt@uk-essen.de

Interessenkonflikt. U. Schara-Schmidt gibt an, dass kein Interessenkonflikt besteht.

Hier steht eine Anzeige.

Springer 